\title{
Erratum to: Predictive role of dynamic contrast enhanced T1-weighted MR sequences in pre-surgical evaluation of macroadenomas consistency
}

\author{
Andrea Romano ${ }^{1,2}$ - Valeria Coppola ${ }^{5}$ - Mariangela Lombardi ${ }^{3}$ Luigi Lavorato ${ }^{4}$. \\ Domenica Di Stefano ${ }^{3}$ Emanuela Caroli $^{4}$ - Maria Camilla Rossi Espagnet ${ }^{1,7}$. \\ Francesca Tavanti $^{1} \cdot$ Giuseppe Minniti $^{6,8} \cdot$ Giuseppe Trillò $^{4} \cdot$ Alessandro Bozzao $^{1}$
}

Published online: 21 November 2016

(C) Springer Science+Business Media New York 2016

\section{Erratum to: Pituitary \\ DOI 10.1007/s11102-016-0760-z}

In the original publication, the coauthor Dr. Giuseppe Minniti's affiliation IRCCS Neuromed affiliation has not been included. The research activity is mainly done at the
Istituto di Ricerca e Cura a Carattere Scientifico (IRCCS) Neuromed, where the coauthor has a research contract.

This erratum is published to correct the affiliation for Dr. Giuseppe Minniti.

The online version of the original article can be found under doi:10.1007/s11102-016-0760-z.

Andrea Romano

andrea.romano@uniroma1.it

1 Department of Neuroradiology, S. Andrea Hospital, University Sapienza, Rome, Italy

2 Department of Odontostomatological and Maxillo-Facial Sciences, Umberto I Hospital, University Sapienza, Rome, Italy

3 Department of Histopathology, S. Andrea Hospital, University Sapienza, Rome, Italy

4 Department of Neurosurgery, S. Andrea Hospital, University Sapienza, Rome, Italy

5 Department of Neuroradiology, S. Carlo Hospital, Potenza, Italy

6 Unit of Radiation Oncology, Sant'Andrea Hospital, University Sapienza, Rome, Italy

7 Neuroradiology Unit, Imaging Department, Bambino Gesù Children's Hospital, Rome, Italy

8 Unit of Radiation Oncology, IRCCS Neuromed, Pozzilli, IS, Italy 